\title{
Controlling the fine-tuning problem with singlet scalar dark matter
}

\author{
Indrani Chakraborty ${ }^{1}$ and Anirban Kundu ${ }^{1}$ \\ ${ }^{1}$ Department of Physics, University of Calcutta, \\ 92, Acharya Prafulla Chandra Road, Kolkata 700009, India.
}

(Dated: August 29, 2018)

\begin{abstract}
Assuming that no other conventional new physics is found immediately at the LHC, we investigate how just the consistent solution of the scalar mass hierarchy problem points towards the minimal necessary field content. We show that to ameliorate the fine-tuning problem, one needs to introduce more scalar degrees of freedom. The simplest solution is one or more real singlets (with the possibility of combining two of them in a complex singlet), which may act as viable cold dark matter candidates, because the constraints on the scalar potential disfavor any mixing between the new scalar(s) with the SM doublet. Furthermore, the fine-tuning problem of the new scalars necessitates the introduction of vector-like fermions. Thus, singlet scalar(s) and vector fermions are minimal enhancements over the Standard Model to alleviate the fine-tuning problem. We also show that the model predicts Landau poles for all the scalar couplings, whose positions depend only on the number of such singlets. Thus, introduction of some new physics at that scale becomes inevitable. We also discuss how the model confronts the LHC constraints and the latest XENON100 data.
\end{abstract}

PACS numbers: 14.80.Ec, 95.35.+d

Keywords: Singlet scalar, Fine-tuning problem, Vector fermions, Dark matter

\section{INTRODUCTION}

Assuming that the Higgs boson has been found at $m_{h}=125.9 \pm 0.4 \mathrm{GeV}$ [1, 2], the Standard Model (SM) is now complete, but there are enough reasons to believe that this is not the final story, rather, at best an effective theory valid up to a certain energy scale. Two such major reasons are the fine-tuning problem of the Higgs boson mass and the existence of the dark matter (DM). One might even add the massive neutrinos.

There are various extensions of the SM to explain the first problem; some of them, like R-parity conserving supersymmetry, Universal extra dimensions, or Little Higgs models with T-parity (i.e. almost any model with a discrete $Z_{2}$ symmetry), also provide us a viable DM candidate. Unfortunately, till now there has been no direct detection in the colliders of any new physics (NP) particle. While there are some interesting hints, there are no compelling indirect evidence too, coming from low-energy observables like those in flavour physics.

Recently, it was pointed out in Refs. [3, 4] that the Higgs boson quartic coupling, $\lambda$, has such a value for $m_{h} \approx 125$ $\mathrm{GeV}$ that it might remain perturbative all the way up to the Planck scale; neither does it blow up and hit the Landau pole, nor does it become negative and make the electroweak vacuum unstable. Thus, there seems to be a distinct possibility that a desert lies between the electroweak scale, parametrized by $v(\approx 246 \mathrm{GeV})$, the vacuum expectation value $(\mathrm{VEV})$ of the Higgs field, and the Planck scale $M_{P l}\left(\sim 10^{19} \mathrm{GeV}\right)$.

In this paper, we would like to investigate whether such a possibility is allowed without running afoul of the finetuning problem, and if not, what can the possible directions of solution be. It was pointed out long ago by Veltman [5] that the fine-tuning problem can be ameliorated if the quadratically divergent term for Higgs self-energy, generated by all the degrees of freedom of the model, is either zero or very small, by some symmetry of the model. For example, all supersymmetric models are only a particular application of this idea, where the contributions from bosonic and fermionic degrees of freedom cancel in the limit of exact supersymmetry.

It is a well-known fact that the SM falls short of the Veltman condition (VC), now that we know all the masses. Thus, there must be some new degrees of freedom lurking somewhere, which interact with the Higgs boson and contribute to its self-energy. If we apply the principle of Occam's razor and focus only on the fine-tuning problem, we will see that the simplest such extension is to introduce one or more scalars; more chiral fermions only worsen the fine-tuning. We will concentrate on gauge singlet scalars, whose phenomenology is well-investigated [6- 10], including the LHC constraints [11], and the stability of the electroweak vacuum [12]. The role of singlet scalars for solving the fine-tuning problem was discussed earlier in Refs. [13, 14] and has recently been investigated in Ref. [15]. It was shown in Ref. [16] that with a large number of singlets, the electroweak phase transition may become a strong first-order one. As is well-known, singlet scalars with a right-sign mass term, and therefore without a VEV, do not mix with the SM doublet, and thus can be good candidates for cold DM (see Section II for details) provided they satisfy other constraints like the DM density or DM-nucleon scattering cross-section limits. However, the fine-tuning problem is not generally addressed in the scalar DM models, assuming that some other mechanism must take care of the same. An exception is Ref. [17], where the interplay of the Veltman condition (for the SM Higgs only) and scalar DM was discussed. We show how the fine-tuning problem can be interlinked with one or more possible DM candidates, treating 
all the Veltman conditions consistently.

We will show in this paper that the solution of the $\mathrm{VC}$ with one or more singlets disfavors the scenario where the singlets mix with the SM doublet. In that case, they can be good cold DM candidates. At the same time, the solution of the $\mathrm{VC}$ for the singlets necessitates the introduction of vectorial fermions. However, they are much less constrained from precision electroweak observables than new chiral fermions (degenerate vector fermions that do not mix with the SM chiral fermions do not contribute to the oblique parameters $S$ and $T$ ) [18], and from the rate of Higgs production via gluon-gluon fusion as they do not couple with the SM Higgs. Also, a new heavy chiral generation would make the Higgs self-coupling $\lambda$ to go negative and make the vacuum unstable, but vector fermions, which are either only singlets or only doublets, do not run into that problem as they couple with the doublet Higgs if and only if they mix with the SM fermions. The role of vector fermions, when they mix with the SM fermions, or when the $125 \mathrm{GeV}$ scalar is an admixture of a doublet and a singlet state, in enhancing the $h \rightarrow \gamma \gamma$ rate has been investigated in the literature [19]. Talking about the diphoton excess, it has been shown that if this anomaly persists, it will point to some new bosonic degrees of freedom at a few $\mathrm{TeV}$ [20]. Furthermore, these fermions can play an important role to make the electroweak phase transition a strong first-order one [21, 22]. However, note that neither the diphoton decay rate nor the evolution of the Higgs quartic coupling is affected if the new vectorial fermions fall in a definite representation of $\mathrm{SU}(2)$ (only singlets or only doublets), and there is no mixing with the SM fermions. Spin- $\frac{1}{2}$ DM candidates and possible allowed effective operators have also received attention [23], but here we do not consider the possibility that the vector fermions are the DM candidates.

We will next concentrate on the evolution of the new couplings with energy. We will show that while the Yukawa couplings of the new fermions remain under control, the scalar self couplings tend to blow up and hit the Landau pole. The exact position of the pole depends on the number of singlets $N$, which is less than $1 \mathrm{TeV}$ for one singlet, about $150 \mathrm{TeV}$ for $N=4$, and more than $10^{4} \mathrm{TeV}$ for $N=10$. Of course, some way before the Landau pole, the couplings cease to be perturbative. If we take this scale as the point of onset of NP, we have every reason to hope that some new degrees of freedom will show up at the upgraded LHC at $\sqrt{s}=14 \mathrm{TeV}$ unless the number of such singlets is not abnormally large. This need not be in the form of the gauge singlet scalars, which will be hard to produce at the LHC, but possibly be in the form of new vector fermions, where the colored fermions can be produced from gluon-gluon or quark-antiquark fusion. However, this statement requires a more careful discussion, to which we will come back later.

Thus, we can rephrase our aim: if one does not see supersymmetry, or extra dimensional models, or any other popular NP scenarios at the LHC, does it necessarily mean the possibility of a barren desert between the electroweak scale and the Planck scale? The answer, as expected, is in the negative, but a spin-off is a possible candidate for cold DM. Solution of the fine-tuning problem hints at bosonic DM, for which the singlet scalars are perhaps the best candidate. We show that a theory with only SM augmented by singlet scalar(s) necessarily mean some NP at the $\mathrm{TeV}$ scale or higher. To put it in another way, if one considers the singlet scalar DM scenario with an eye to the fine-tuning problem of the scalar masses, there exists a solution but only with the introduction of vectorial fermions; this is the minimal enhancement over the SM that is necessary.

The paper is arranged as follows. In Section II, we discuss the scalar potential and the corresponding Veltman conditions. In Section III, we study the renormalization group (RG) evolution of the couplings and its possible consequences. We summarize and conclude in Section IV.

\section{THE VELTMAN CONDITION}

Let us first consider the SM augmented with one real singlet scalar $S$. The potential is

$$
V(\Phi, S)=V_{\mathrm{SM}}+V_{\text {singlet }}=-\mu^{2} \Phi^{\dagger} \Phi+\lambda\left(\Phi^{\dagger} \Phi\right)^{2}-M^{2} S^{2}+\tilde{\lambda} S^{4}+a S^{2}\left(\Phi^{\dagger} \Phi\right),
$$

where $\Phi$ is the SM doublet, with $\langle\Phi\rangle=v / \sqrt{2}$. We will take both $\mu^{2}, M^{2}>0$ to start with, and denote the SM Higgs boson, the remnant of $\Phi$ after spontaneous symmetry breaking, by $h$. There might be a cubic term $c S^{3}$ in the potential, but that would not affect the subsequent analysis. The linear terms in $S$ giving rise to tadpole diagrams are assumed to cancel out and they will remain so even after the quantum corrections. This happens if we take the tadpole potential to be [ 6 ]

$$
V_{\text {tadpole }}=\alpha_{1} S+\alpha_{2} \Phi^{\dagger} \Phi S
$$

with $\alpha_{1}+\frac{1}{2} \alpha_{2} v^{2}=0$. There might also be a discrete symmetry, like $S \rightarrow-S$, preventing odd terms. For $N$ singlets with an $O(N)$ symmetry, the potential looks like

$$
V\left(\Phi, S_{i}\right)=-\mu^{2} \Phi^{\dagger} \Phi+\lambda\left(\Phi^{\dagger} \Phi\right)^{2}-M^{2} \sum_{i} S_{i}^{2}+\tilde{\lambda}\left(\sum_{i} S_{i}^{2}\right)^{2}+a\left(\Phi^{\dagger} \Phi\right) \sum_{i} S_{i}^{2} .
$$


With only the SM, i.e., $a=0$, the Higgs self-energy receives a quadratically divergent correction

$$
\delta m_{h}^{2}=\frac{\Lambda^{2}}{16 \pi^{2}}\left(6 \lambda+\frac{3}{4} g_{1}^{2}+\frac{9}{4} g_{2}^{2}-6 g_{t}^{2}\right),
$$

where $g_{1}$ and $g_{2}$ are the $U(1)_{Y}$ and $S U(2)_{L}$ gauge couplings, and $g_{t}=\sqrt{2} m_{t} / v$ is the top quark Yukawa coupling. We treat all other fermions as massless, and use the cut-off regularization, $\Lambda$ being the cutoff scale. Note that while this regularization is not Lorentz invariant, this has the nice feature of separating the quadratic and the logarithmic divergences. Other methods, such as dimensional regularization, do not discriminate between these two divergences, and we get a slightly different correction [24], which includes both quadratic and logarithmic divergences:

$$
\delta m_{h}^{2} \propto \frac{1}{\epsilon}\left(6 \lambda+\frac{1}{4} g_{1}^{2}+\frac{3}{4} g_{2}^{2}-6 g_{t}^{2}\right) .
$$

As our goal is to cancel the strongest divergence, we will use the cut-off regularization.

The Veltman condition (VC) demands that the quantity inside parentheses in Eq. (4) be made zero, or at least controllably small, by some symmetry. There are further quadratic divergences coming from two-loop diagrams, but they are suppressed from one-loop contributions by a factor of $\ln (\Lambda / \mu) / 16 \pi^{2}$, where $\mu$ is the regularization scale, and is in general under control.

One can say that the quadratic divergence is under control if $\left|\delta m_{h}^{2}\right| \leq m_{h}^{2}$, which translates into

$$
\left|m_{h}^{2}+2 m_{W}^{2}+m_{Z}^{2}-4 m_{t}^{2}\right| \leq \frac{16 \pi^{2}}{3} \frac{v^{2}}{\Lambda^{2}} m_{h}^{2} .
$$

This inequality is clearly not satisfied in the SM for $v^{2} / \Lambda^{2} \leq 0.1$, or $\Lambda \geq 760 \mathrm{GeV}$, and onset of NP at such a low scale is almost ruled out by the LHC. Any more chiral fermions will only make the left-hand side of Eq. (6) more negative and hence worsen the fine-tuning problem. What is needed is a positive contribution, and extra scalars are a viable option.

With one extra singlet $S$, as in Eq. (1), the $\mathrm{VC}$ is modified to

$$
\delta m_{h}^{2}=\frac{\Lambda^{2}}{16 \pi^{2}}\left(6 \lambda+\frac{3}{4} g_{1}^{2}+\frac{9}{4} g_{2}^{2}-6 g_{t}^{2}+a\right)
$$

and with $N$ number of identical singlets (i.e., an $O(N)$ symmetric singlet sector), the last term is replaced by $N a$.

For $N=1$, we find that $a=4.17$, which is quite large even if not nonperturbative (we take $4 \pi \approx 12.56$ to be the threshold for nonperturbativity to set in). More singlets bring down the value to $4.17 / N$. This is to be taken as an indicative value only, as there is no reason why this value would be absolutely stable if we take higher-order corrections (for an estimate of higher-order effects, we refer the reader to [15], where it can be seen that such corrections bring a marginal change.) To be consistent, we will limit our discussions within one-loop effects only, and therefore use only one-loop renormalization group $(\mathrm{RG})$ equations to calculate the evolution of the couplings.

We need to consider the VC for the singlet too. The condition does not depend on whether the singlet develops a VEV or not. This reads

$$
\delta m_{S}^{2}=\frac{\Lambda^{2}}{16 \pi^{2}}[(8+4 N) \tilde{\lambda}+4 a] .
$$

So, only with singlet, we need a large (and definitely nonperturbative) and negative quartic coupling, and the potential develops a minimum unbounded from below in the direction $|\Phi|=$ constant and $|S| \rightarrow \infty$. Thus, this solution is clearly unacceptable.

While one needs some negative contribution to Eq. (8), one notes that this cannot come from chiral fermions of the SM as they do not couple to $S$. Thus, one is led to introduce vector fermions - either singlets or doublets under $S U(2)$. This introduces further terms in the potential:

$$
\mathcal{L}_{V F}=-m_{F} \bar{F} F-\zeta_{F} \bar{F} F S,
$$

and the mass of $F$ is $m_{F}+\zeta_{F}\langle S\rangle$. Note that a symmetry like $S \rightarrow-S$ implies $F \rightarrow i \gamma_{5} F$ and hence forbids the bare mass term, unless the symmetry is explicitly broken. We will not pursue this possibility further as we show explicitly that a nonzero VEV to the singlet is disfavored, so the vector fermions must get their masses from the bare mass term. For this case, i.e., $M^{2}<0$ and $\langle S\rangle=0$, there is no mass term from the Yukawa couplings. Direct searches at the LHC put a lower limit of the order of $500 \mathrm{GeV}$ on the mass of vector quarks. 
For simplicity, we assume a complete generation of vector fermions $(N, E),(U, D)$ with all Yukawa couplings $\zeta_{i}$ to be the same (the heavy neutrino $N$ is not to be confused with $N$, the number of singlets). One can, in principle, consider only one such fermion in the spectrum, or only the lepton or quark doublet. The VC for $S$ now reads

$$
\delta m_{S}^{2}=\frac{\Lambda^{2}}{16 \pi^{2}}\left[(8+4 N) \tilde{\lambda}+4 a-4 Z^{2}\right]
$$

where

$$
Z^{2}=\sum_{i} N_{c} \zeta_{i}^{2}=\zeta_{E}^{2}+\zeta_{N}^{2}+3\left(\zeta_{U}^{2}+\zeta_{D}^{2}\right)=8 \zeta^{2}
$$

$N_{c}$ being the color of the corresponding fermions. While this does not guarantee a degenerate generation, that remains a distinct possibility, and thus one can avoid the strong constraints coming from oblique $S$ and $T$ parameters-because of the vectorial nature and degeneracy.

If there is only one singlet and $M^{2}>0$, the minimization conditions are

$$
-\mu^{2}+\lambda v^{2}+a v^{\prime 2}=0, \quad-M^{2}+2 \tilde{\lambda} v^{\prime 2}+\frac{1}{2} a v^{2}=0,
$$

where $\langle S\rangle=v^{\prime}$. The mass term can be written as

$$
\left(\begin{array}{ll}
h & S
\end{array}\right) \mathcal{M}\left(\begin{array}{c}
h \\
S
\end{array}\right)=\left(\begin{array}{ll}
h & S
\end{array}\right)\left(\begin{array}{cc}
\lambda v^{2} & a v v^{\prime} \\
a v v^{\prime} & 4 \tilde{\lambda} v^{\prime 2}
\end{array}\right)\left(\begin{array}{l}
h \\
S
\end{array}\right) .
$$

The condition for both masses to be real is

$$
4 \lambda \tilde{\lambda} \geq a^{2} .
$$

For $\lambda \approx 0.13$ and $a \approx 4$, this makes $\tilde{\lambda}$ very large $(\geq 33)$ and clearly nonperturbative. While this by itself may still be acceptable, the fact that all the scalar couplings hit their respective Landau poles almost right at the electroweak scale rules this option out.

One might wonder whether the situation improves in the large- $N$ limit. However, if the original scalar sector has an $O(N)$ symmetry, a spontaneous symmetry breaking will result in $N-1$ Goldstone bosons, which couple to the doublet Higgs $h$, and therefore will give a very large invisible decay width of $h$. This is again unacceptable from the measurement of the branching fractions of the Higgs at the LHC.

Thus, we are forced to take $M^{2}<0$, so that the singlet does not develop a VEV and there is no singlet-doublet mixing. This is true even if there are more than one such singlets. The mass-squared of the singlet is given by

$$
m_{S}^{2}=2 M^{2}+a v^{2},
$$

so for $N=1$, the lowest possible mass for the singlet is about $500 \mathrm{GeV}$, and goes down as $1 / \sqrt{N}$. This means no change in the decay pattern of the $125 \mathrm{GeV}$ scalar from the SM Higgs, and no $h \rightarrow S S$ invisible decay unless $N$ is so large that $m_{S}<m_{h} / 2$ (this happens for $N \geq 66$ ). Also, there are no changes to the oblique parameters $S, T$, and $U$ [6].

\section{A. The Cosmological Constraints}

Although the mass of the singlet(s) has a lower limit, it is nevertheless a free parameter of the theory. The number of singlets $N$ is another free parameter, but the singlet-doublet coupling strength $a$ is not (assuming an $O(N)$ symmetric singlet sector). The $O(N)$ symmetry might be broken softly and by a very small amount to make the lightest scalar nondegenerate. The vector fermions have only a marginal influence on the viability of this singlet as a DM candidate if $m_{F}>m_{S}$, which we will assume to be true in our entire analysis.

Over almost the entire parameter space relevant for our discussion $\left(100 \mathrm{GeV}<m_{S}<800 \mathrm{GeV}, 0.1<a<4.2\right)$, the singlet density is below the DM relic density [25]

$$
\Omega_{D M} h^{2}=0.1138 \pm 0.0045,
$$

except for high $m_{S}$ and very low $a$, so there is no conflict with overclosure of the universe. The singlet can scatter off the nucleons, by exchange of the Higgs boson; it can be a tree-level scattering off the quarks or a one-loop scattering 


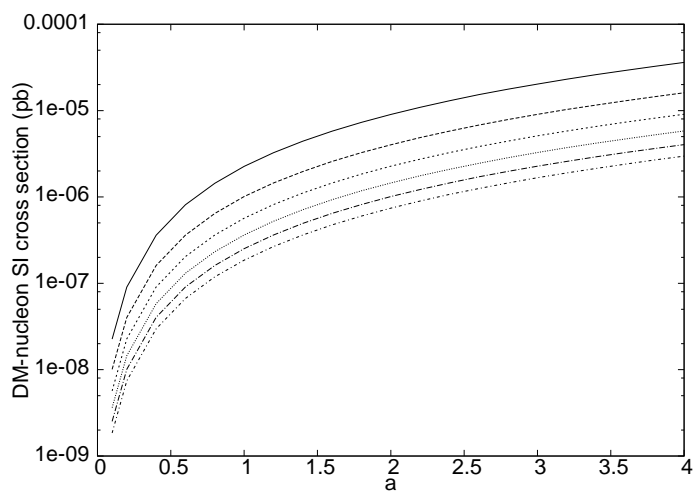

FIG. 1: The spin-independent dark matter-nucleon cross section as a function of the singlet-doublet coupling parameter $a$. The lines, from top to bottom, are for the singlet mass $m_{S}=200,300,400,500,600$ and $700 \mathrm{GeV}$ respectively. Even for $N$ such singlets, we have assumed a slight nondegeneracy (see text) so that only one of the singlets is the DM candidate.

off the gluons. The cross-section is necessarily spin-independent, which is rather strongly bounded by the data from 225 days running of XENON100 [26]. Roughly, the $2 \sigma$ upper bound for spin-independent DM-nucleon scattering is of the order of $2-4 \times 10^{-8} \mathrm{pb}$, for the DM mass between 300 and $1000 \mathrm{GeV}$. We have checked through the software micrOMEGAs [27] that this bound is satisfied only for small couplings $\left(a \leq 0.3-0.5\right.$, depending on $\left.m_{S}\right)$, see Fig. 1 for details. This is easy to understand as the cross-section grows as $a^{2}$. This might mean that for a successful solution of the fine-tuning problem, we need a large number of scalars $N \sim 10$, leading to the possibility of a strong first-order electroweak phase transition. However, around a mass window of $65-100 \mathrm{GeV}$, where $h \rightarrow S S$ would still be forbidden, there is an allowed window with a much larger DM-nucleon spin-independent cross-section found by Dama/I [28] (this is in apparent conflict with XENON100), where one can have larger values of $a$ and hence a smaller number of singlets.

\section{B. The vector fermions}

The vector fermions also merit a brief discussion. First, let us note that they have to be vectorial because (i) they must couple to the singlet, and (ii) heavy chiral fermions are highly constrained from the LHC data, and they drive the SM Higgs quartic coupling $\lambda$ to negative values and hence make the electroweak vacuum unstable. The vector fermions can be $S U(2)$ singlets or doublets; the only difference is their couplings to the electroweak gauge bosons.

Second, We have assumed a complete generation of $(N, E)$ and $(U, D)$, but one can work with partial generations too. In fact, just a single vector neutrino would have been sufficient to solve the Veltman condition for the singlet scalar, albeit with a much higher Yukawa coupling. If all the vector fermions are exactly degenerate (which is not necessary for the model, but just a simplifying assumption) stationary states, they would have been stable, which is in conflict with cosmological bounds on stable charged and/or colored particles. A possible way out would be a very small admixture of the vector fermions, at least the lightest lepton and quark if we admit a nondegeneracy, with the chiral fermions. With such a tiny admixture, the vector fermions will be stable on the collider scale but not on the cosmological scale. At the same time, none of the flavor observables would be significantly affected. The scalar $S$ can be a DM candidate if $m_{S}<m_{F}$, which we assume in our analysis.

The vector quarks can be produced at the LHC by $g g$ or $q \bar{q}$ fusion, just like a heavy sequential quark. They will leave a jetty track by radiating off gluons, and will ultimately dump their energy in the hadron calorimeter. In fact, if they are stable on the collider scale (which is a necessity if the singlet scalar has to be a DM candidate - otherwise the scalar will decay to lighter SM states through virtual vector fermions), they should form bound states with ordinary quarks. LHC experiments have searched for such long-lived quarks or leptons [29], and the mass limits depend on the quantum numbers of them. The lower limit for the mass of a long-lived lepton is of the order of 350-400 GeV, and that of a quark which can form a bound state is of the order of $700 \mathrm{GeV}$. If they are close to the mass limits, LHC at $\sqrt{s}=14 \mathrm{TeV}$ might be able to produce them. The vector fermions must also be almost degenerate so as not to affect the oblique parameters.

One should also note that ultraheavy fermions run the risk of having their Yukawa couplings hitting the Landau pole [30]. However, here the mass does not come from the Yukawa couplings but from the bare mass terms, so such a constraint is not applicable here. 


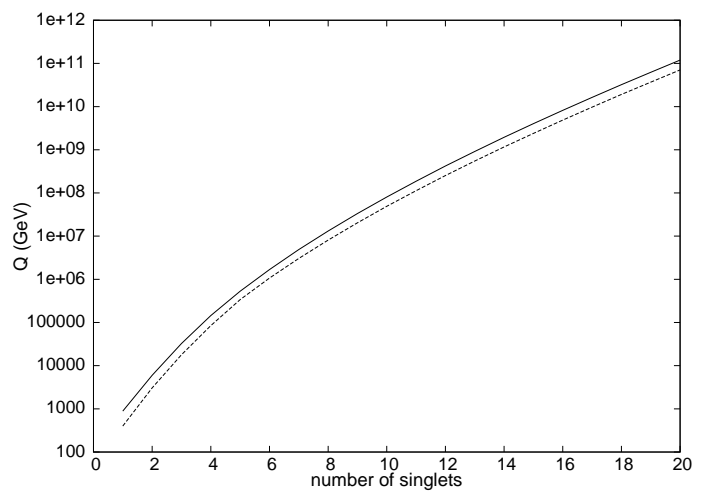

FIG. 2: The energy scales where (i) the scalar quartic couplings hit the Landau poles (assuming one-loop RG equations to be still valid) [upper curve] and (ii) at least one of the scalar couplings ceases to be perturbative $(\leq 4 \pi)$ [lower curve].

\section{THE ONE-LOOP RG EQUATIONS}

Next, we would like to see how the couplings evolve with energy. The one-loop $\beta$-functions are

$$
\begin{aligned}
16 \pi^{2} \beta_{\lambda} & =12 \lambda^{2}+6 g_{t}^{2} \lambda+N a^{2}-\frac{3}{2} \lambda\left(g_{1}^{2}+3 g_{2}^{2}\right)-3 g_{t}^{4}+\frac{3}{16}\left(g_{1}^{4}+2 g_{1}^{2} g_{2}^{2}+3 g_{2}^{4}\right), \\
16 \pi^{2} \beta_{\tilde{\lambda}} & =(32+4 N) \tilde{\lambda}^{2}+a^{2}+4 \tilde{\lambda} Z^{2}-\sum_{i} N_{c} \zeta_{i}^{4} \\
16 \pi^{2} \beta_{a} & =\left[6 \lambda+12 \tilde{\lambda}+4 a+6 g_{t}^{2}+4 Z^{2}-\frac{3}{2} g_{1}^{2}-\frac{9}{2} g_{2}^{2}\right] a \\
16 \pi^{2} \beta_{g_{t}} & =\left[\frac{9}{4} g_{t}^{2}-\frac{17}{24} g_{1}^{2}-\frac{9}{8} g_{2}^{2}-4 g_{3}^{2}\right] g_{t} \\
16 \pi^{2} \beta_{g_{3}} & =-\frac{17}{6} g_{3}^{3} \theta\left(Q^{2}-m_{U}^{2}\right)-\frac{19}{6} g_{3}^{3} \theta\left(m_{U}^{2}-Q^{2}\right) \\
16 \pi^{2} \beta_{\zeta_{U}} & =\left[\frac{3}{2} \zeta_{U}^{2}+Z^{2}-\frac{4}{3}\left(\frac{1}{12}\right) g_{1}^{2}-0\left(\frac{9}{4}\right) g_{2}^{2}-4 g_{3}^{2}\right] \zeta_{U} \\
16 \pi^{2} \beta_{\zeta_{D}} & =\left[\frac{3}{2} \zeta_{U}^{2}+Z^{2}-\frac{1}{3}\left(\frac{1}{12}\right) g_{1}^{2}-0\left(\frac{9}{4}\right) g_{2}^{2}-4 g_{3}^{2}\right] \zeta_{D} \\
16 \pi^{2} \beta_{\zeta_{N}} & =\left[\frac{3}{2} \zeta_{N}^{2}+Z^{2}-0\left(\frac{3}{4}\right) g_{1}^{2}-0\left(\frac{9}{4}\right) g_{2}^{2}\right] \zeta_{N} \\
16 \pi^{2} \beta_{\zeta_{E}} & =\left[\frac{3}{2} \zeta_{E}^{2}+Z^{2}-3\left(\frac{3}{4}\right) g_{1}^{2}-0\left(\frac{9}{4}\right) g_{2}^{2}\right] \zeta_{E}
\end{aligned}
$$

where $\beta_{h} \equiv d h / d t$, and $t \equiv \ln \left(Q^{2} / \mu^{2}\right)$, and we have taken all vector fermions to be heavier than the top. Note that our definition of $t$ differs by a factor of 2 from that used by some authors. For the new fermions, the $\beta$-functions are given for the singlet (doublet) type vectors. For simplicity, we have put all the SM Yukawa couplings equal to zero except for that of the top quark. This hardly changes our conclusions.

The main feature of the above equations that all the three scalar quartic couplings, namely, $\lambda, \tilde{\lambda}$, and $a$, simultaneously hit their Landau poles; this is because of the way their RG equations are coupled. We start with $\tilde{\lambda}\left(Q^{2}=m_{Z}^{2}\right)=0$, but the result is insensitive to the precise boundary condition. The other boundary conditions are fixed by the Higgs boson mass and the Veltman conditions. The singlet Yukawa couplings $\zeta_{i}$ remain perturbative for the entire domain. In fact, the DM phenomenology, like the DM-nucleon cross-section, does not depend on the precise value of $\zeta_{i}$, which are fixed only by the singlet Veltman condition.

It might appear counterintuitive that with the increase in $N$, the number of singlets, the couplings blow up at a slower rate. However, note that the initial value of $a$ depends on $N$; while $a^{2}\left(Q^{2}=m_{Z}^{2}\right)$ goes down as $1 / N^{2}$, the contribution to $\beta_{\lambda}$ increases only as $N$, so the blow-up is slower.

Again, one must not read too much in the exact position of the Landau pole for at least two reasons: first, we do not know as yet how stable the Veltman conditions are if we take higher-order quantum corrections, and second, one should not use perturbative $\beta$-functions when at least one of the couplings become large. While we have no control over the first issue, except dimensional arguments that the higher-order corrections should be small, the second point 
is taken care of if we focus not only on the Landau poles but the points where at least one of the couplings goes nonperturbative, i.e., above $4 \pi$. As expected, this scale is somewhat below the Landau pole, as can be seen in Fig. 22, Note that the gauge quantum numbers of the vector fermions are of no consequence for this plot, as is evident from Eq. (17).

The singlet scalar DM interacts with the SM particles (excluding the vector fermions) only through the exchange of the SM Higgs boson $h[6]$, either by $g g \rightarrow h \rightarrow S S$ or $q \bar{q} \rightarrow h \rightarrow S S$. Thus, the DM-nucleon scattering cross-section is entirely spin-independent. Note that the sensitivity for detection goes down with $m_{S}$.

Further tweaks on the model are possible. For example, one can think of mixing between vector and chiral fermions, consistent with the flavor physics observables. This might open up a decay channel for the singlet(s), like $S \rightarrow f \bar{f}$ (where the stationary state $f$ is a mixture of vector and chiral states), and spoil the candidature of the singlet for DM. This, however, does not invalidate our main conclusion of the necessity of singlet scalar(s) and vector fermion(s) for a successful solution of the fine-tuning problem. The mixing, of course, can be adjusted to be so tiny as to make the singlet(s) stable on a cosmological time scale, but this smells of fine-tuning and goes against the philosophy of this article, so we do not recommend this solution. One can also do away with the quark doublet altogether, and just think of a lepton doublet $(N, E)$, with $m_{E}>m_{N}$, so that $N$ can either be stable or decay to SM leptons through charged-current processes. Even a single heavy neutrino can do the job of solving the fine-tuning problem of the singlet, and in fact will be welcome because the decay $S \rightarrow \gamma \gamma$ through a heavy fermion loop will be automatically absent. It is also possible to build a multicomponent singlet model without the $O(N)$ symmetry, but impose further discrete symmetries so that one or more singlets do not couple with the vector fermions but the rest do.

The singlets can even be produced at the LHC, either by gluon-gluon fusion through a vector fermion loop, or through radiation off a vector quark. For the former process, one might tag with an associated monojet or single photon; for the second, there will be a missing energy signature. However, the rates are expected to be quite small for such massive scalars, even at the LHC upgrade.

\section{SUMMARY}

We have shown how the fine-tuning problem and the existence of cold dark matter can be linked if one assumes an underlying but yet-to-be-discovered symmetry that cancels, or at least softens, the quadratic divergence of scalar masses. While the cancellation of quadratic divergences for the SM Higgs can be performed by more scalar degrees of freedom, like one or more singlets, the same for the singlets necessitates the introduction of vector fermions. Thus, the minimal enhancement of the SM that can successfully address both the fine-tuning problem and the existence of cold DM is one with one or more singlet scalars that do not mix with the SM doublet, and vector fermions, either singlets or doublets under $S U(2)$.

This model is internally consistent too. The singlet scalars do not mix with the SM doublet because (i) their self-coupling becomes so large and nonperturbative that the theory hits the Landau pole at the electroweak scale itself; (ii) for more than one singlets with an $O(N)$ symmetry, there are $N-1$ Goldstones in the theory, resulting in a large invisible decay width of the SM Higgs. The vector fermions do not couple with the SM Higgs, but the stationary states, in particular the third generation states, might contain a small admixture of vectorial fermions unless prohibited by some discrete symmetry. Such vector fermions and singlet scalars also successfully evade the constraints coming from the oblique parameters.

With these new terms in the potential, the scalar couplings evolve in a different way. With more positive contributions in the respective $\beta$-functions, all the scalar quartic couplings blow up simultaneously somewhere below the Planck scale. The exact position of the Landau pole depends on the number of singlet scalars; for one scalar, this is even below $1 \mathrm{TeV}$, so a consistent picture should have at least two real singlets (or one complex singlet). Thus, there must be some new physics beyond that point, which may very well be responsible for the symmetry. In fact, if the Landau pole is at about $10-100 \mathrm{TeV}$, the fine-tuning problem is never that severe, maybe only one or two orders of magnitude higher than what we have for supersymmetric models, but still it will be aesthetically pleasing to have a way out of the problem.

There are at least two free parameters of the model apart from those fixed by the Veltman conditions: the singlet scalar mass and the vector fermion mass. While the scalar mass can be constrained by the relic density of the DM and the DM-nucleon scattering cross section, the only constraint on the fermion masses comes from non-observability at the LHC. A way to rule this model out might be a nonzero spin-dependent cross-section in DM-nucleon scattering. However, we emphasize again that the motivation for this model is to supply a minimal extension of the SM to successfully address the hierarchy problem, and the appearance of a scalar DM candidate should only be taken as a spin-off. 


\section{Acknowledgments}

IC was supported by a research fellowship of CSIR, Government of India. AK was supported by CSIR, Government of India (project no. 03(1135)/9/EMR-II), and also by the DRS programme of the UGC, Government of India.

[1] S. Chatrchyan et al. [CMS Collaboration], Phys. Lett. B 716, 30 (2012) arXiv:1207.7235] [hep-ex]].

[2] G. Aad et al. [ATLAS Collaboration], Phys. Lett. B 716, 1 (2012) arXiv:1207.7214 [hep-ex]]; G. Aad et al. [ATLAS Collaboration], Phys. Rev. D 86, 032003 (2012) arXiv:1207.0319 [hep-ex]].

[3] G. Degrassi, S. Di Vita, J. Elias-Miro, J. R. Espinosa, G. F. Giudice, G. Isidori and A. Strumia, JHEP 1208, 098 (2012) arXiv:1205.6497 [hep-ph]].

[4] S. Alekhin, A. Djouadi and S. Moch, Phys. Lett. B 716, 214 (2012) arXiv:1207.0980 [hep-ph]].

[5] M. J. G. Veltman, Acta Phys. Polon. B 12, 437 (1981).

[6] V. Barger, P. Langacker, M. McCaskey, M. J. Ramsey-Musolf and G. Shaughnessy, Phys. Rev. D 77, 035005 (2008) arXiv:0706.4311 [hep-ph]]. For phenomenology of singlet scalars, see Ref. [2-12] of this.

[7] V. Barger, P. Langacker, M. McCaskey, M. Ramsey-Musolf and G. Shaughnessy, Phys. Rev. D 79, 015018 (2009) arXiv:0811.0393 [hep-ph]].

[8] B. Batell, D. McKeen and M. Pospelov, JHEP 1210, 104 (2012) arXiv:1207.6252 [hep-ph]].

[9] M. Gonderinger, H. Lim and M. J. Ramsey-Musolf, Phys. Rev. D 86, 043511 (2012) [arXiv:1202.1316] [hep-ph]]; G. Belanger, K. Kannike, A. Pukhov and M. Raidal, arXiv:1211.1014 [hep-ph].

[10] V. Silveira and A. Zee, Phys. Lett. B 161, 136 (1985); J. McDonald, Phys. Rev. D 50, 3637 (1994) hep-ph/0702143; C. P. Burgess, M. Pospelov and T. ter Veldhuis, Nucl. Phys. B 619, 709 (2001) hep-ph/0011335; W. -L. Guo and Y. -L. Wu, JHEP 1010, 083 (2010) arXiv:1006.2518 [hep-ph]]. A. Biswas and D. Majumdar, arXiv:1102.3024 [hep-ph]; S. Bar-Shalom, M. Geller, S. Nandi and A. Soni, arXiv:1208.3195 [hep-ph]; S. Baek, P. Ko, W. -I. Park and E. Senaha, JHEP 1211, 116 (2012) arXiv:1209.4163 [hep-ph]]; J. M. Cline and K. Kainulainen, arXiv:1210.4196 [hep-ph].

[11] A. Djouadi, O. Lebedev, Y. Mambrini and J. Quevillon, Phys. Lett. B 709 (2012) 65 arXiv:1112.3299 [hep-ph]].

[12] M. Kadastik, K. Kannike, A. Racioppi and M. Raidal, JHEP 1205, 061 (2012) arXiv:1112.3647] [hep-ph]].

[13] A. Kundu and S. Raychaudhuri, Phys. Rev. D 53, 4042 (1996) hep-ph/9410291.

[14] B. Grzadkowski and J. Wudka, Phys. Rev. Lett. 103, 091802 (2009) arXiv:0902.0628 [hep-ph]].

[15] A. Drozd, B. Grzadkowski and J. Wudka, JHEP 1204, 006 (2012) arXiv:1112.2582 [hep-ph]].

[16] O. Bahat-Treidel, Y. Grossman and Y. Rozen, JHEP 0705, 022 (2007) hep-ph/0611162 ; J. R. Espinosa and M. Quiros, Phys. Rev. D 76, 076004 (2007) hep-ph/0701145.

[17] F. Bazzocchi, M. Fabbrichesi and P. Ullio, Phys. Rev. D 75, 056004 (2007) hep-ph/0612280]; F. Bazzocchi and M. Fabbrichesi, arXiv:1207.0951 [hep-ph].

[18] L. Lavoura and J. P. Silva, Phys. Rev. D 47, 2046 (1993); G. Cynolter and E. Lendvai, Eur. Phys. J. C 58, 463 (2008) arXiv:0804.4080 [hep-ph]].

[19] B. Batell, S. Jung and H. M. Lee, arXiv:1211.2449 [hep-ph]; S. Dawson, E. Furlan and I. Lewis, arXiv:1210.6663 [hep-ph].

[20] N. Arkani-Hamed, K. Blum, R. T. D'Agnolo and J. Fan, arXiv:1207.4482 [hep-ph].

[21] M. S. Carena, A. Megevand, M. Quiros and C. E. M. Wagner, Nucl. Phys. B 716, 319 (2005) hep-ph/0410352.

[22] H. Davoudiasl, I. Lewis and E. Ponton, arXiv:1211.3449 [hep-ph].

[23] K. Cheung, P. -Y. Tseng, Y. -L. S. Tsai and T. -C. Yuan, JCAP 1205, 001 (2012) arXiv:1201.3402 [hep-ph]].

[24] M. B. Einhorn and D. R. T. Jones, Phys. Rev. D 46, 5206 (1992).

[25] G. Hinshaw, D. Larson, E. Komatsu, D. N. Spergel, C. L. Bennett, J. Dunkley, M. R. Nolta and M. Halpern et al., arXiv:1212.5226 [astro-ph.CO].

[26] E. Aprile et al. [XENON100 Collaboration], Phys. Rev. Lett. 109, 181301 (2012) arXiv:1207.5988 [astro-ph.CO]].

[27] G. Belanger, F. Boudjema, P. Brun, A. Pukhov, S. Rosier-Lees, P. Salati and A. Semenov, Comput. Phys. Commun. 182, 842 (2011) arXiv:1004.1092 [hep-ph]].

[28] C. Savage, G. Gelmini, P. Gondolo and K. Freese, JCAP 0904, 010 (2009) arXiv:0808.3607 [astro-ph]].

[29] See, e.g. V. Khachatryan et al. [CMS Collaboration], JHEP 1103, 024 (2011) arXiv:1101.1645 [hep-ex]]; G. Aad et al. [ATLAS Collaboration], arXiv:1211.1597 [hep-ex].

[30] M. S. Chanowitz, M. A. Furman and I. Hinchliffe, Phys. Lett. B 78, 285 (1978), Nucl. Phys. B 153, 402 (1979). 